TRANSACTIONS OF THE

AMERICAN MATHEMATICAL SOCIETY

Volume 353, Number 1, Pages 291-311

S 0002-9947(00)02568-X

Article electronically published on September 18, 2000

\title{
THE NUMBER OF PLANAR CENTRAL CONFIGURATIONS IS FINITE WHEN $N-1$ MASS POSITIONS ARE FIXED
}

\author{
PETER W. LINDSTROM
}

\begin{abstract}
In this paper, it is proved that for $n>2$ and $n \neq 4$, if $n-1$ masses are located at fixed points in a plane, then there are only a finite number of $n$-point central configurations that can be generated by positioning a given additional $n$th mass in the same plane. The result is established by proving an equivalent isolation result for planar central configurations of five or more points. Other general properties of central configurations are established in the process. These relate to the amount of centrality lost when a point mass is perturbed and to derivatives associated with central configurations.
\end{abstract}

\section{INTRODUCTION}

Central configurations are initial positions of masses that lead to special families of solutions of the $n$-body problem. If the masses are released with zero velocity from a central configuration, the particles move toward the center of gravity in such a way that the configuration collapses homothetically to a collision singularity. Planar central configurations with the appropriate initial velocities can also give rise to other special solutions where the particles travel in elliptical orbits and the configuration remains similar to the initial configuration throughout the motion. For these and other reasons central configurations have been studied extensively. See Moeckel [5] and Saari [8] for detailed introductions to the subject.

Results pertaining to how many central configurations exist have appeared over the time period from Euler [2, Lagrange [3, and Moulton [7] to the present. Smale [9] recently described eighteen great problems for the next century. Sixth in his list, assembled at the request of V. I. Arnold on behalf of the International Mathematical Union, is the question of whether, for each set of $n$ positive masses, the number of nonequivalent planar central configurations is finite. The present paper was motivated by embedding this question in a sequence of successively more difficult ones. Making use of Definition 2.4 it is seen that Smale's sixth problem is equivalent to the last $(k=n-2)$ in the following sequence of finiteness questions.

Finiteness Questions (FQ). For $k=1,2, \ldots, n-2$, if $n-k$ positive masses are located at fixed points in a plane, is it always true that only a finite number of n-point central configurations can be generated by positioning $k$ additional given positive masses in the same plane?

The answer to his question is known for $n=3$, but not for any greater value. In this case, if two mass locations are fixed, there are exactly five three-point planar

Received by the editors December 18, 1998.

2000 Mathematics Subject Classification. Primary 70F10.

(C)2000 American Mathematical Society 
central configurations that can be generated by positioning the third mass in the same plane. Three of these are collinear and the other two are in the form of an equilateral triangle. For $n=4$, a partial answer has been obtained by Moeckel [6]. He proved that the set of mass 4-tuples for which the answer is "no" must have measure zero. For $n>3$, it appears that the answers to FQ were also unknown for all values of $k$ less than $n-2$. In this paper we give an affirmative answer when $k=1$ and $n$ is greater than 4 .

In the process of proving this result we also prove some general theorems related to derivatives and derivative matrices that are associated with planar central configurations.

\section{Definitions AND STRATEGy}

Planar central configurations arise in the context of the planar Newtonian $n$ body problem which deals with the motion of $n$ particles (point-masses) in the plane whose motion is governed by Newton's second law via the forces determined by the Newtonian potential.

Let the mass of the $j$ th particle located at $X_{j}=\left[\begin{array}{l}x_{j} \\ y_{j}\end{array}\right]$ be denoted by $m_{j}>$ 0 . The motion is governed by $m_{j} \ddot{X}_{j}=\left[\begin{array}{c}\frac{\partial U}{\partial x_{j}} \\ \frac{\partial U}{\partial y_{j}}\end{array}\right]$, where $U \equiv U\left(X_{1}, X_{2}, \ldots, X_{n}\right)=$ $\sum_{\substack{i, j) \\ i<j}} \frac{m_{i} m_{j}}{\left|X_{i}-X_{j}\right|}$ is the Newtonian potential.

For convenience we will assume $\sum_{j=1}^{n} m_{j}=1$, and will denote $\ddot{X}_{j}$, the acceleration of the $j$ th particle, by $A_{j}$, and the center of mass by $C_{M}=\left[\begin{array}{l}\sum_{j=1}^{n} m_{j} x_{j} \\ \sum_{j=1}^{n} m_{j} y_{j}\end{array}\right]$. $Q_{j}=C_{M}-X_{j}$ will denote the vector from $X_{j}$ to $C_{M}$.

Definition 2.1. Notation. A planar configuration $G=\left\{X_{1}, X_{2}, \ldots, X_{n} ; m_{1}, m_{2}\right.$, $\left.\ldots, m_{n}\right\}$ of $n$ point masses is a choice of distinct positions $X_{1}, X_{2}, \ldots, X_{n} \in R^{2}$ and positive masses $m_{1}, m_{2}, \ldots, m_{n} \in R^{1}$.

Definition 2.2. A planar configuration $G$ is called a central configuration, if there is a positive number $\lambda$, such that for $j=1, \ldots, n, \frac{1}{m_{j}}\left[\begin{array}{c}\frac{\partial U}{\partial x_{j}} \\ \frac{\partial U}{\partial y_{j}}\end{array}\right]=\lambda Q_{j}$.

From Newton's second law it follows that a planar configuration is central if there exists a positive $\lambda$ such that for $j=1, \ldots, n, A_{j}=\lambda Q_{j}$. It can be shown that if $\lambda$ exists, then it is given by $\lambda=\frac{U}{I}$, where $I=\sum_{j=1}^{n} m_{j}\left|Q_{j}\right|^{2}$, is the moment of inertia of the configuration about the center of mass.

Definition 2.3. For the planar configuration $\left\{X_{1}, X_{2}, \ldots, X_{n} ; m_{1}, m_{2}, \ldots, m_{n}\right\}$, we say that the point-mass located at $X_{k}$ possesses the centrality property if $A_{k}-$ $\lambda Q_{k}=0$ where $\lambda=\frac{U}{I}$.

Using this definition we can describe a planar central configuration as one in which each point-mass possesses the centrality property.

As far as the $n$-body problem is concerned there is no need to distinguish between planar central configurations that differ by translation or rotation. Also, if they differ only by scale it is sensible to view them as equivalent because under a homothetic collapse the larger would collapse through the smaller. Hence equivalence of planar central configurations is defined as follows:

Definition 2.4. Two planar central configurations are equivalent, if it is true that when both are translated so that each has the origin as its center of mass, then 
one can be transformed into the other by an expansion or contraction of the plane followed by a rotation about the origin.

An obvious strategy for showing that only a finite number of planar central configurations can be generated by augmenting a given $n-1$ point configuration with an additional mass located at a variable point $\left[\begin{array}{l}x_{n} \\ y_{n}\end{array}\right]$ is to view the $n$ vector equations defining the central configuration as representing $2 n$ curves in an $\left(x_{n}, y_{n}\right)$ plane, and then to try and directly show that there are only a finite number of points at which all the curves intersect. The intersection points of the curves can be shown to be intersection points of curves represented by high degree polynomials in the variables $x_{n}, y_{n}$. So it must be shown that generally these curves have no component in common. This direct approach appears to be quite difficult given the freedom in assigning mass values and in choosing the fixed $n-1$ mass locations.

Our approach, though less complete, fails only in the case $n=4$. Basically we use linearization and establish an equivalent "one-point" isolation result for planar central configurations of five or more points. We show that if the positions of all but one of the masses in a planar central configuration are held fixed, then there is a neighborhood of the position of the remaining $n$th mass, such that if this mass is moved to some other position in that neighborhood, then the resulting configuration is no longer central.

The isolation result is developed using

$$
D_{j n}=\left[\begin{array}{ll}
\frac{\partial p_{j 1}}{\partial x_{n}} & \frac{\partial p_{j 1}}{\partial y_{n}} \\
\frac{\partial p_{j 2}}{\partial x_{n}} & \frac{\partial p_{j 2}}{\partial y_{n}}
\end{array}\right],
$$

where $P_{j}=A_{j}-\lambda Q_{j}=\left[\begin{array}{c}p_{j 1} \\ p_{j 2}\end{array}\right]$ and $P_{j}$ is viewed as a function of $X_{n}$. Having a particular form for this derivative matrix, the isolation result follows quickly in the case when the mass to be perturbed is situated at the center of mass. In the other cases, the configuration is rescaled and repositioned so that the mass to be perturbed is situated at the origin and the center of mass is at $(1,0)$. It is then shown that were the isolation result false, the other $n-1$ points of the configuration, via the singularity of the matrices $D_{j n}, j=1, \ldots, n-1$, would reside on a planar curve (we call it the singularity locus) determined solely by $I$ and $\lambda$. In addition, null spaces, associated with each of these points on the singularity locus, would have a nonzero intersection. The crux of the paper lies in the proof that such a nonzero intersection is impossible when $n>4$.

Although it may if the masses are suitably restricted, the interesting case of $n=4$, does not generally yield to our approach. The fact that it does suggests further investigation of four-point central configurations having one mass at the origin and the other three on a singularity locus.

In the final section of this paper, we use the results that were developed to answer the finiteness question to prove that a slight perturbation in the position of any single point-mass in a planar central configuration of more than 4 points destroys the centrality property for at least $n-4$ of the nonperturbed masses.

\section{The Derivative of $P_{j}=A_{j}-\lambda Q_{j}$ at a Central configuration}

In this section, we fix $X_{1}, X_{2}, \ldots, X_{n-1}$ and $m_{1}, m_{2}, \ldots, m_{n}$ and for notational clarity use $Z=\left[\begin{array}{l}z_{1} \\ z_{2}\end{array}\right]$ to represent the variable $n$th mass position. Thus we will be working with the configuration $G(Z)=\left\{X_{1}, X_{2}, \ldots, X_{n-1}, Z ; m_{1}, m_{2}, \ldots, m_{n}\right\}$. 
$X_{n}$ will be reserved to represent a position $Z$ for which $G(Z)$ is a central configuration.

We view $P_{j}, j=1, \ldots, n-1 ; I, U, \lambda=\frac{U}{I}$, and $C_{M}$ as functions of the variable point $Z$, and use $F^{\prime}(Z)$ to denote the $k \times 2$ Jacobian matrix of a function $F: R^{2} \rightarrow$ $R^{k}$. $A(Z)$ will denote the acceleration of the mass at $Z$, and $Q(Z)=C_{M}(Z)-Z$, the vector from $Z$ to the center of mass. Our objective is to find a useful expression that characterizes the Jacobian matrix of $P_{j}$ when $Z=X_{n}$. To avoid confusion with the moment of inertia, the $2 \times 2$ identity matrix will be denoted by $E$.

Lemma 3.1. $I^{\prime}(Z)=-2 m_{n} Q^{T}(Z)$.

Proof. On writing $C_{M}(Z)=\left[\begin{array}{l}c_{1}\left(z_{1}\right) \\ c_{2}\left(z_{2}\right)\end{array}\right]$, we have

$$
\begin{array}{r}
I(Z)=\sum_{j=1}^{n-1} m_{j}\left[\left(x_{j}-c_{1}\left(z_{1}\right)\right)^{2}+\left(y_{j}-c_{2}\left(z_{2}\right)\right)^{2}\right] \\
+m_{n}\left[\left(z_{1}-c_{1}\left(z_{1}\right)\right)^{2}+\left(z_{2}-c_{2}\left(z_{2}\right)\right)^{2}\right] .
\end{array}
$$

Using $c_{1}^{\prime}\left(z_{1}\right)=m_{n}$ gives

$$
\begin{aligned}
\frac{\partial I}{\partial z_{1}} & =-2 m_{n}\left[\sum_{j=1}^{n-1} m_{j}\left[\left(x_{j}-c_{1}\left(z_{1}\right)\right)\right]+m_{n}\left(z_{1}-c_{1}\left(z_{1}\right)\right)\right]+2 m_{n}\left(z_{1}-c_{1}\left(z_{1}\right)\right) \\
& =2 m_{n}\left(z_{1}-c_{1}\left(z_{1}\right)\right)
\end{aligned}
$$

Similarly, $\frac{\partial I}{\partial z_{2}}=2 m_{n}\left(z_{2}-c_{2}\left(z_{2}\right)\right)$. Thus

$$
I^{\prime}(Z)=-2 m_{n}\left[\left(c_{1}\left(z_{1}\right)-z_{1}\right),\left(c_{2}\left(z_{2}\right)-z_{2}\right)\right]=-2 m_{n} Q^{T}(Z) .
$$

Lemma 3.2. When $Z=X_{n}$ and the configuration $G(Z)$ is central, $\lambda^{\prime}(Z)=$ $\frac{3 m_{n} \lambda(Z)}{I(Z)} Q^{T}(Z)$.

Proof. $\lambda^{\prime}(Z)=\frac{I(Z) U^{\prime}(Z)-U(Z) I^{\prime}(Z)}{I^{2}(Z)}$. Since $U^{\prime}(Z)=m_{n} A^{T}(Z)$ and $A(Z)=\lambda(Z) Q(Z)$ when $Z=X_{n}$,

$$
\begin{aligned}
\lambda^{\prime}(Z) & =\frac{I(Z) U^{\prime}(Z)-U(Z) I^{\prime}(Z)}{I^{2}(Z)}=\frac{m_{n} \lambda(Z)}{I(Z)} Q^{T}(Z)+\frac{2 m_{n} U(Z)}{I^{2}(Z)} Q^{T}(Z) \\
& =\frac{3 m_{n} \lambda(Z)}{I(Z)} Q^{T}(Z) .
\end{aligned}
$$

In the next lemma, $V_{j}(Z)=X_{j}-Z, j=1, \ldots, n-1$, will denote the vector from $Z$ to $X_{j}$, and, for $T \in R^{2}, H_{j}(T)$ will denote the representation in the usual basis of the projection of $T$ on $V_{j}(Z)$. Also, we will make use of the following:

i) If $Y(Z)=g(Z) F(Z)$, where $g: R^{2} \rightarrow R^{1}$ and $F: R^{2} \rightarrow R^{2}$, then for $T \in$ $R^{2} Y^{\prime}(Z) T=g(Z)\left\{\left[F^{\prime}(Z)\right] T\right\}+\left\{\left[g^{\prime}(Z)\right] T\right\} F(Z)$.

ii) $V_{j}^{\prime}(Z)=-E$.

iii) $\left(\left|V_{j}(Z)\right|^{k}\right)^{\prime}=-k\left|V_{j}(Z)\right|^{k-2} V_{j}^{T}(Z)$.

Lemma 3.3. For $T \in R^{2}$ and $j=1,2, \ldots, n-1, A_{j}^{\prime}(Z) T=\frac{m_{n}}{\left|V_{j}(Z)\right|^{3}}\left(T-3 H_{j}(T)\right)$. 
Proof. $A_{j}(Z)=W-m_{n}\left|V_{j}(Z)\right|^{-3} V_{j}(Z)$ where $W$ is constant. Thus, using i), ii), and iii) with $g(Z)=\left|V_{j}(Z)\right|^{-3}$ and $F(Z)=V_{j}(Z)$, we have

$$
\begin{aligned}
A_{j}^{\prime}(Z) T & =-m_{n}\left(-\left|V_{j}(Z)\right|^{-3} T+\left\{3\left|V_{j}(Z)\right|^{-5} V_{j}^{T}(Z) T\right\} V_{j}(Z)\right) \\
& =\frac{m_{n}}{\left|V_{j}(Z)\right|^{3}}\left(T-3 H_{j}(T)\right) .
\end{aligned}
$$

Let $B_{j}(Z)=\lambda(Z) Q_{j}(Z)$.

Lemma 3.4. For $T \in R^{2}$, when $Z=X_{n}$ and the configuration $G(Z)$ is central, $B_{j}^{\prime}(Z) T=m_{n} \lambda(Z)\left(T+\frac{3}{I(Z)}\left(Q^{T}(Z) T\right) Q_{j}(Z)\right), j=1,2, \ldots, n-1$.

Proof. Using $Q_{j}^{\prime}(Z)=m_{n} E$, Lemma 3.2, and i) with $g(Z)=\lambda(Z)$ and $F(Z)=$ $Q_{j}(Z)$ yields

$$
\begin{aligned}
B_{j}^{\prime}(Z) T & =\lambda(Z)\left\{m_{n} T\right\}+\left\{\frac{3 m_{n} \lambda(Z)}{I(Z)} Q^{T}(Z) T\right\} Q_{j}(Z) \\
& =m_{n} \lambda(Z)\left(T+\frac{3}{I(Z)}\left(Q^{T}(Z) T\right) Q_{j}(Z)\right) .
\end{aligned}
$$

Proposition 3.1. For $T \in R^{2}$, when $Z=X_{n}$ and the configuration $G(Z)$ is central,

$$
\begin{aligned}
P_{j}^{\prime}(Z) T=\frac{-3 m_{n}}{\left|V_{j}(Z)\right|^{3}\left\{H_{j}(T)+\frac{\lambda(Z)\left|V_{j}(Z)\right|^{3}}{I(Z)}\left(Q^{T}(Z) T\right) Q_{j}(Z)\right.} \\
\left.-\left(\frac{1-\lambda(Z)\left|V_{j}(Z)\right|^{3}}{3}\right) T\right\},
\end{aligned}
$$

$j=1,2, \ldots, n-1$.

Proof. Lemmas 3.3 and 3.4 yield

$$
\begin{aligned}
& P_{j}^{\prime}(Z) T= \frac{m_{n}}{\left|V_{j}(Z)\right|^{3}}\left(T-3 H_{j}(T)\right)-m_{n} \lambda(Z)\left(T+\frac{3}{I(Z)}\left(Q^{T}(Z) T\right) Q_{j}(Z)\right) \\
&=\frac{-3 m_{n}}{\left|V_{j}(Z)\right|^{3}}\left\{H_{j}(T)+\frac{\lambda(Z)\left|V_{j}(Z)\right|^{3}}{I(Z)}\left(Q^{T}(Z) T\right) Q_{j}(Z)\right. \\
&\left.-\left(\frac{1-\lambda(Z)\left|V_{j}(Z)\right|^{3}}{3}\right) T\right\} .
\end{aligned}
$$

4. One-point isolation: The distinguished point at the Center of mass

For $n>3$, we show that if $X_{n}$ is the center of mass of the planar central configuration $G\left(X_{n}\right)$, then there is a neighborhood of $X_{n}$, such that if $Z \neq X_{n}$ is in the neighborhood, then $G(Z)$ is not central.

Proposition 4.1. If $G\left(X_{n}\right)$ is a planar central configuration and $\left\{G\left(Z_{k}\right)\right\}$ is a sequence of planar central configurations for which $Z_{k} \neq X_{n}$ and $Z_{k} \rightarrow X_{n}$, then there is a unit vector $T \in R^{2}$, independent of $j$, such that for $j=1, \ldots, n-1$, $P_{j}^{\prime}\left(X_{n}\right) T=0$.

Proof. Let $\Delta Z_{k}=Z_{k}-X_{n}$. With no loss it can be assumed that $\frac{\Delta Z_{k}}{\left|\Delta Z_{k}\right|} \rightarrow T$. Now since $P_{j}\left(X_{n}+\Delta Z_{k}\right)=P_{j}\left(X_{n}\right)=0, P_{j}^{\prime}\left(X_{n}\right) \frac{\Delta Z_{k}}{\left|\Delta Z_{k}\right|}=\frac{P_{j}\left(X_{n}+\Delta Z_{k}\right)-P_{j}\left(X_{n}\right)}{\left|\Delta Z_{k}\right|}+\frac{o\left(\Delta Z_{k}\right)}{\left|\Delta Z_{k}\right|}=$ $\frac{o\left(\Delta Z_{k}\right)}{\left|\Delta Z_{k}\right|} \rightarrow 0$. Thus, in the limit, $P_{j}^{\prime}\left(X_{n}\right) T=0$. 
Theorem 4.1. If $X_{n}$ is the center of mass of the planar central configuration $G\left(X_{n}\right)$, then for any unit vector $T \in R^{2}$ there exist at most two distinct values of $j$ less than $n$ for which $P_{j}^{\prime}\left(X_{n}\right) T=0$.

Proof. Since $X_{n}$ is the center of mass, $Q\left(X_{n}\right)=0$. Thus if $j<n$ and $P_{j}^{\prime}\left(X_{n}\right) T=0$, it follows from Proposition 3.1 that

$$
\left\{H_{j}(T)-\left(\frac{1-\lambda\left(X_{n}\right)\left|V_{j}\left(X_{n}\right)\right|^{3}}{3}\right) T\right\}=0 .
$$

Thus, $\frac{1-\lambda\left(X_{n}\right)\left|V_{j}\left(X_{n}\right)\right|^{3}}{3}$ is an eigenvalue of the projection operator corresponding to $H_{j}$, and hence is 0 or 1 . Since $\frac{1-\lambda\left(X_{n}\right)\left|V_{j}\left(X_{n}\right)\right|^{3}}{3}<1$, it must equal 0 . Hence $X_{j}$ is on a circle of radius $\sqrt[3]{\frac{1}{\lambda\left(X_{n}\right)}}$ centered at $X_{n}$. Additionally, the radius vector $V_{j}\left(X_{n}\right)$ from $X_{n}$ to $X_{j}$ is perpendicular to $T$. The result follows from the fact that there are only two possible positions on the circle for $X_{j}$ for which $T$ can be perpendicular to $V_{j}\left(X_{n}\right)$.

The next corollary is used in the final section to establish a general property of directional derivatives related to central configurations. For this purpose a change of notation is useful. Only for this corollary, Corollary 5.1, and then as described in the last section we will use the following assumptions and notation.

Definition 4.1. Notation and assumptions for $n$ variable particles. It will be assumed that $X_{1}, X_{2}, \ldots, X_{n}$ are variable positions of $n$ particles in a plane having positive masses $m_{1}, m_{2}, \ldots, m_{n}$. $X$ will denote the point

$$
\left[\begin{array}{c}
X_{1} \\
X_{2} \\
\vdots \\
X_{n}
\end{array}\right]
$$

in $R^{2 n}$, and $X^{0}$ will equal

$$
\left[\begin{array}{c}
X_{10} \\
X_{20} \\
\vdots \\
X_{n 0}
\end{array}\right]
$$

where $X_{i 0}$ is a particular position of the $i$ th particle. $\lambda, A_{j}, Q_{j}$, and $P_{j} ; j=$ $1,2, \ldots, n$, will be viewed as functions of $X$, and $D P_{j}\left(X^{0}\right)$ will denote the $2 \times 2 n$ derivative matrix of $P_{j}(X)$ evaluated at $X^{0}$. For any unit vector $T \in R^{2}, T_{j}^{0}$ will denote

$$
\left[\begin{array}{c}
X_{10} \\
X_{20} \\
\vdots \\
X_{j 0}+T \\
\vdots \\
X_{n 0}
\end{array}\right]-X^{0}
$$

Corollary 4.1. If $X_{n 0}$ is the center of mass of the planar central configuration $\left\{X_{10}, X_{20}, \ldots, X_{n 0} ; m_{1}, m_{2}, \ldots, m_{n}\right\}$, then for any unit vector $T \in R^{2}$, there exists at most two distinct values of $j, 1 \leq j<n$, for which $\operatorname{Lim}_{h \rightarrow 0^{+}} \frac{\left|P_{j}\left(X^{0}+h T_{n}^{0}\right)\right|}{h}=0$. 
Proof. Since $P_{j}\left(X^{0}\right)=0, \operatorname{Lim}_{h \rightarrow 0^{+}} \frac{\left|P_{j}\left(X^{0}+h T_{n}^{0}\right)\right|}{h}=0$ yields $D P_{j}\left(X^{0}\right) T_{n}^{0}=0$. In the notation used prior to this corollary this means $P_{j}^{\prime}\left(X_{n 0}\right) T=0$. With $G\left(X_{n}\right)$ replaced by $\left\{X_{10}, X_{20}, \ldots, X_{n 0} ; m_{1}, m_{2}, \ldots, m_{n}\right\}$, the result follows from Theorem 4.1 .

Theorem 4.2. If $n>3$ and $X_{n}$ is the center of mass of the planar central configuration $G\left(X_{n}\right)$, then there is a neighborhood of $X_{n}$ such that if $Z \neq X_{n}$ is in the neighborhood, then $G(Z)$ is not central.

Proof. If such a neighborhood did not exist, there would be a sequence of planar central configurations $\left\{G\left(Z_{k}\right)\right\}$ for which $Z_{k} \neq X_{n}$ and $Z_{k} \rightarrow X_{n}$. From Proposition 4.1, there would then exist a unit vector $T \in R^{2}$ for which $P_{j}^{\prime}\left(X_{n}\right) T=0$, $j=1,2, \ldots, n-1$, and this would contradict Theorem 4.1.

\section{One-Point isolation: The Zero-one case}

In this section we assume the central configuration $G\left(X_{n}\right)$ is in "zero-one" form, meaning that the distinguished point $X_{n}$ is located at the origin and the center of mass of the configuration is one unit to the right at $(1,0)$. Thus in proving isolation for a zero-one central configuration, we will be taking $Z$ near 0 . Note that $V_{j}(0)$ is the vector from 0 to $\left[\begin{array}{l}x_{j} \\ y_{j}\end{array}\right]$. We will therefore denote its length by $r_{j}=\sqrt{x_{j}^{2}+y_{j}^{2}}$. Also, for conciseness of notation, we will use $\lambda$ in place of $\lambda(0)$, and $I$ in place of $I(0)$. We begin by obtaining the matrix $P_{j}^{\prime}(Z)$ when $Z=0$.

Proposition 5.1. For the planar central configuration $G(0)$, with center of mass at $(1,0)$,

$$
P_{j}^{\prime}(0)=\frac{-3 m_{n}}{r_{j}^{3}}\left[\begin{array}{cc}
\frac{x_{j}^{2}}{r_{j}^{2}}+\frac{\lambda r_{j}^{3}\left(1-x_{j}\right)}{I}-\frac{1-\lambda r_{j}^{3}}{3} & \frac{x_{j} y_{j}}{r_{j}^{2}} \\
\frac{x_{j} y_{j}}{r_{j}^{2}}-\frac{\lambda r_{j}^{3}}{I} y_{j} & \frac{y_{j}^{2}}{r_{j}^{2}}-\frac{1-\lambda r_{j}^{3}}{3}
\end{array}\right] .
$$

Proof. We note that $V_{j}(0)=X_{j}$ and hence that $H_{j}(T)$ is the projection of $T$ on $X_{j}$. Thus from Proposition 3.1,

$$
P_{j}^{\prime}(0)\left[\begin{array}{l}
1 \\
0
\end{array}\right]=\frac{-3 m_{n}}{r_{j}^{3}}\left\{\frac{1}{r_{j}^{2}}\left[\begin{array}{c}
x_{j}^{2} \\
x_{j} y_{j}
\end{array}\right]+\frac{\lambda r_{j}^{3}}{I}\left(Q^{T}(0)\left[\begin{array}{l}
1 \\
0
\end{array}\right]\right) Q_{j}(0)-\left(\frac{1-\lambda r_{j}^{3}}{3}\right)\left[\begin{array}{l}
1 \\
0
\end{array}\right]\right\} .
$$

Using $Q_{j}(0)=\left[\begin{array}{c}1-x_{j} \\ -y_{j}\end{array}\right]$ and $Q(0)=\left[\begin{array}{l}1 \\ 0\end{array}\right]$ gives

$$
P_{j}^{\prime}(0)\left[\begin{array}{l}
1 \\
0
\end{array}\right]=\frac{-3 m_{n}}{r_{j}^{3}}\left[\begin{array}{c}
\frac{x_{j}^{2}}{r_{j}^{2}}+\frac{\lambda r_{j}^{3}\left(1-x_{j}\right)}{I}-\frac{1-\lambda r_{j}^{3}}{3} \\
\frac{x_{j} y_{j}}{r_{j}^{2}}-\frac{\lambda r_{j}^{3}}{I} y_{j}
\end{array}\right]
$$

Also,

$$
\begin{aligned}
P_{j}^{\prime}(0)\left[\begin{array}{l}
0 \\
1
\end{array}\right] & =\frac{-3 m_{n}}{r_{j}^{3}}\left\{\frac{1}{r_{j}^{2}}\left[\begin{array}{c}
x_{j} y_{j} \\
y_{j}^{2}
\end{array}\right]+\frac{\lambda r_{j}^{3}}{I}\left(Q^{T}(0)\left[\begin{array}{l}
0 \\
1
\end{array}\right]\right) Q_{j}(0)-\left(\frac{1-\lambda r_{j}^{3}}{3}\right)\left[\begin{array}{l}
0 \\
1
\end{array}\right]\right\} \\
& =\frac{-3 m_{n}}{r_{j}^{3}}\left[\begin{array}{c}
\frac{x_{j} y_{j}}{r_{j}^{2}} \\
\frac{y_{j}^{2}}{r_{j}^{2}}-\frac{1-\lambda r_{j}^{3}}{3}
\end{array}\right],
\end{aligned}
$$

and the result follows. 
According to Proposition 4.1, if one-point isolation were not to hold when $G\left(X_{n}\right)$ is in zero-one form, then $P_{j}^{\prime}(0)$ would be singular. For this reason we examine the matrix that appears in the previous proposition with the goal of gaining insight into its null space as a function of $X=\left[\begin{array}{l}x \\ y\end{array}\right], I$, and $\lambda$. We'll let

$$
S(X ; \lambda, I)=\left[\begin{array}{cc}
\frac{x^{2}}{r^{2}}+\frac{\lambda r^{3}(1-x)}{I}-\frac{1-\lambda r^{3}}{3} & \frac{x y}{r^{2}} \\
\frac{x y}{r^{2}}-\frac{\lambda r^{3}}{I} y & \frac{y^{2}}{r^{2}}-\frac{1-\lambda r^{3}}{3}
\end{array}\right], \text { where } r=\sqrt{x^{2}+y^{2}} .
$$

$N(X ; \lambda, I)$ will denote the null space of $S(X ; \lambda, I)$.

Definition 5.1. The singularity locus is the set of nonzero $X$ for which $S(X ; \lambda, I)$ is singular.

The next proposition gives a polar representation of the singularity locus. When the point $\left[\begin{array}{l}x \\ y\end{array}\right]$ is represented in polar form the angle measured from the positive $x$ axis will be denoted by $\phi$.

Proposition 5.2. The singularity locus is symmetric with respect to the $x$ axis and equal to the set of points whose polar coordinates, $(r, \phi)$, satisfy $-3 \lambda^{2} \cos (\phi) r^{7}+$ $(I+3) \lambda^{2} r^{6}+3 \lambda \cos (\phi) r^{4}+\left(6+I-9 \cos ^{2}(\phi)\right) \lambda r^{3}-2 I=0$, where $r>0$, and $0 \leq \phi<2 \pi$.

Proof. The singularity locus is determined by

$$
\operatorname{Det}\left[\begin{array}{cc}
\frac{x^{2}}{r^{2}}+\frac{\lambda r^{3}(1-x)}{I}-\frac{1-\lambda r^{3}}{3} & \frac{x y}{r^{2}} \\
\frac{x y}{r^{2}}-\frac{\lambda r^{3}}{I} y & \frac{y^{2}}{r^{2}}-\frac{1-\lambda r^{3}}{3}
\end{array}\right]=0 .
$$

Symmetry with respect to the $x$ axis follows from the fact that the value of the determinant is unchanged when $y$ is replaced by $-y$.

On expanding, multiplying through by $9 I$, and using $x=r \cos \phi, y=r \sin \phi$, $\frac{x^{2}}{r^{2}}=\cos ^{2} \phi$, and $\frac{x y}{r^{2}}=\sin \phi \cos \phi$, we get $-3 \lambda^{2} \cos (\phi) r^{7}+(I+3) \lambda^{2} r^{6}+3 \lambda \cos (\phi) r^{4}+$ $\left(6+I-9 \cos ^{2}(\phi)\right) \lambda r^{3}-2 I=0$.

With one exception, the null space of $S(X ; \lambda, I)$ is one dimensional. The exception, as proved in the next lemma, occurs when $\lambda=\frac{1}{(I+1)^{3}}$. In this case, illustrated by Figure 5.1 there is exactly one point on the singularity locus at which it is not one dimensional. The point is

$$
\left[\begin{array}{c}
\sqrt[3]{\frac{1}{\lambda}} \\
0
\end{array}\right]
$$

which is the linking point of components which, as illustrated by Figure [5.2, are unconnected when $\lambda \neq \frac{1}{(I+1)^{3}}$. It should be noted that Figures 5.1 and 5.2 are incomplete in that the component on the right is unbounded and can be shown to have $x=1+\frac{I}{3}$ as a vertical asymptote. We also note that the elliptical portion of the locus is not symmetric with respect to the $y$ axis.

Lemma 5.1. If $X$ is on the singularity locus, $N(X ; \lambda, I)=R^{2}$ if and only if $\lambda=\frac{1}{(I+1)^{3}}$ and $X=\left[\begin{array}{c}I+1 \\ 0\end{array}\right]=\left[\begin{array}{c}\sqrt[3]{\frac{1}{\lambda}} \\ 0\end{array}\right]$.

Proof. If $\lambda=\frac{1}{(I+1)^{3}}$ and $X=\left[\begin{array}{c}I+1 \\ 0\end{array}\right]$, computation gives $S(X ; \lambda, I)=0$. Conversely, if $S(X ; \lambda, I)=0$, then since the upper right entry is 0 , either $x$ or $y$ must be 0 . But if $x$ is $0, y$ must be 0 in order that the lower left entry is 0 . As a consequence, since $X$ cannot equal $\left[\begin{array}{l}0 \\ 0\end{array}\right]$, we must have $y=0$. Further, since the lower right entry 


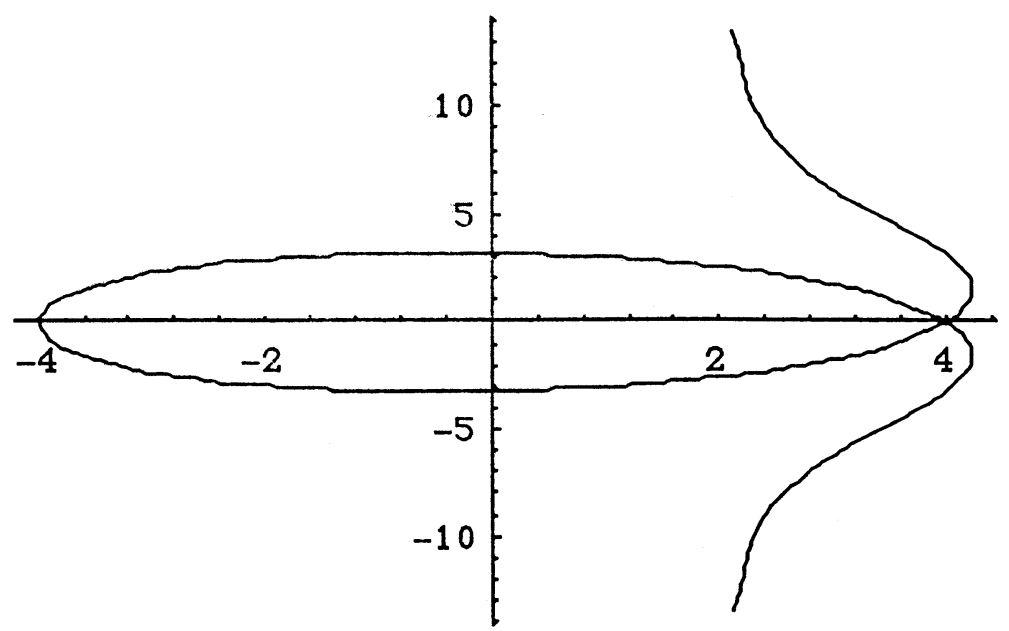

FIgURE 5.1. The singularity locus for $\lambda=\frac{1}{64}, I=3$

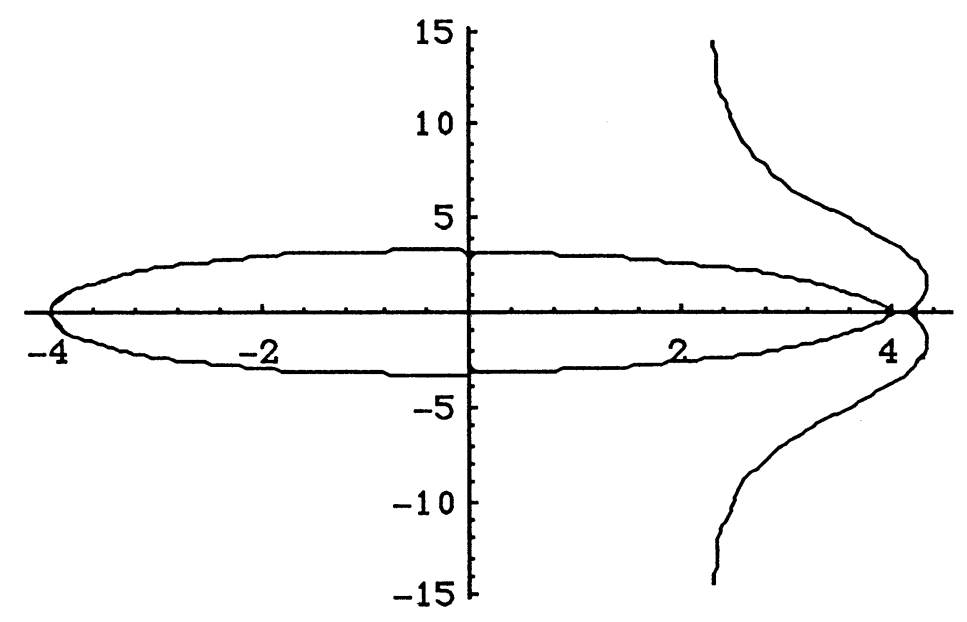

Figure 5.2. The singularity locus for $\lambda=\frac{1}{64}, I=3.5$

is $0, \lambda r^{3}=1$. Then, since $\frac{x^{2}}{r^{2}}=1$, and the upper left is $0, x$ must equal $I+1$, and so, $\lambda=\frac{1}{r^{3}}=\frac{1}{x^{3}}=\frac{1}{(I+1)^{3}}$.

The characterization of $N(X ; \lambda, I)$ will depend upon whether $X$ is on the $x$ axis, the $y$ axis, or on neither axis.

Lemma 5.2. i) If $\lambda \neq \frac{1}{(I+1)^{3}}$, there are exactly three points on the singularity locus corresponding to $y=0$. They are $\left[\begin{array}{c} \pm \sqrt[3]{\frac{1}{\lambda}} \\ 0\end{array}\right]$ and $\left[\begin{array}{c}\eta(\lambda, I) \\ 0\end{array}\right]$, where $\eta(\lambda, I)$ is the positive root of the polynomial $\zeta(r ; \lambda, I)=\frac{\lambda}{I} r^{4}-\lambda\left(\frac{1}{I}+\frac{1}{3}\right) r^{3}-\frac{2}{3}$.

If $\lambda=\frac{1}{(I+1)^{3}}$, then $\left[\begin{array}{c}\sqrt[3]{\frac{1}{\lambda}} \\ 0\end{array}\right]$ and $\left[\begin{array}{c}\eta(\lambda, I) \\ 0\end{array}\right]$ are identical, and there are exactly two points on the singularity locus corresponding to $y=0$. 
ii) There are exactly two points on the singularity locus corresponding to $x=0$. They are $\left[ \pm \frac{0}{\frac{1}{\lambda\left(\frac{I}{3+1}\right)}}\right]$.

Proof. i) When $y=0, \operatorname{Det}(S(X ; \lambda, I))=\left(1+\frac{\lambda r^{3}(1-x)}{I}-\frac{1-\lambda r^{3}}{3}\right)\left(-\frac{1-\lambda r^{3}}{3}\right)=$ $\left(\frac{1-\lambda r^{3}}{3}\right)\left(\frac{\lambda r^{3} x}{I}-\left\{\frac{1}{I}+\frac{1}{3}\right\} \lambda r^{3}-\frac{2}{3}\right)$. Since $x$ can equal $\pm r$, the first factor makes the determinant zero when $x=\left[ \pm \sqrt[3]{\frac{1}{\lambda}}\right]$. While the second factor is nonzero (for positive $r$ ) when $x=-r$, and it is zero when $x=r$, where $r$ is the sole positie root of $\zeta(r ; \lambda, I)$. This root is equal to $\sqrt[3]{\frac{1}{\lambda}}$ if and only if $0=\zeta\left(\sqrt[3]{\frac{1}{\lambda}} ; \lambda, I\right)=$ $\frac{\lambda}{I}\left(\frac{1}{\lambda}\right)^{4 / 3}-\lambda\left(\frac{1}{I}+\frac{1}{3}\right)\left(\frac{1}{\lambda}\right)-\frac{2}{3}=\frac{1}{I}\left(\sqrt[3]{\frac{1}{\lambda}}-1-I\right)$, which is the case if and only if $\lambda=\frac{1}{(I+1)^{3}}$.

ii) When $x=0$,

$$
\begin{aligned}
\operatorname{Det}(S(X ; \lambda, I)) & =\left[\frac{\lambda r^{3}}{I}-\left(\frac{1-\lambda r^{3}}{3}\right)\right]\left[\frac{2+\lambda r^{3}}{3}\right] \\
& =\frac{1}{3}\left[\lambda r^{3}\left(\frac{3+I}{I}\right)-1\right]\left[\frac{2+\lambda r^{3}}{3}\right] .
\end{aligned}
$$

The only $y$ for which the determinant equals zero are those for which $\lambda r^{3}=\frac{I}{3+I}$. This gives $y= \pm \sqrt[3]{\frac{1}{\lambda}\left(\frac{I}{3+I}\right)}$.

When $X$ is on the singularity locus and $N(X ; \lambda, I) \neq R^{2}, K(X ; \lambda, I)$ will denote the slope of the line that constitutes $N(X ; \lambda, I)$.

Proposition 5.3. Let $x=\left[\begin{array}{l}x \\ y\end{array}\right]$ be on the singularity locus. Then

i) if $x \neq 0$ and $y \neq 0, K(X ; \lambda, I)$ is finite and given by

$$
K(X ; \lambda, I)=\frac{y}{x}\left[\frac{3+I}{I}\right]\left[\frac{\left(\frac{I}{3+I}\right)-\lambda r^{3}}{1-\lambda r^{3}}\right] .
$$

ii) if $x=0, K(X ; \lambda, I)=\frac{y}{2+I}$, where $y= \pm \sqrt[3]{\frac{1}{\lambda}\left(\frac{I}{3+I}\right)}$.

iii) if $x=-\sqrt[3]{\frac{1}{\lambda}}$ and $y=0, N(X ; \lambda, I)$ is spanned by $\left[\begin{array}{l}0 \\ 1\end{array}\right]$, and $K(X ; \lambda, I)$ is undefined.

iv) if $x=\sqrt[3]{\frac{1}{\lambda}}, y=0$, and $\lambda \neq \frac{1}{(I+1)^{3}}, N(X ; \lambda, I)$ is spanned by $\left[\begin{array}{l}0 \\ 1\end{array}\right]$, and $K(X ; \lambda, I)$ is undefined.

$\mathrm{v})$ if $x=\eta(\lambda, I), y=0$, and $\lambda \neq \frac{1}{(I+1)^{3}}$, (where $\eta(\lambda, I)$ is as defined in Lemma 5.2), $K(X ; \lambda, I)=0$.

Proof. i) Assume $X=\left[\begin{array}{l}x \\ y\end{array}\right]$ is on the singularity locus, $x \neq 0$ and $y \neq 0$. From Lemma 5.1 $y \neq 0$ implies $N(X ; \lambda, I) \neq R^{2}$. Multiplying the bottom row of $S(X ; \lambda, I)$ by $\frac{x}{y}$ and subtracting the result from the top row yields the matrix

$$
S_{1}(X ; \lambda, I)=\left[\begin{array}{ll}
\frac{\lambda r^{3}}{I}-\frac{1-\lambda r^{3}}{3} & \frac{x}{y}\left(\frac{1-\lambda r^{3}}{3}\right) \\
\frac{x y}{r^{2}}-\frac{\lambda r^{3}}{I} y & \frac{y^{2}}{r^{2}}-\frac{1-\lambda r^{3}}{3}
\end{array}\right] .
$$

If $\lambda r^{3}=1$, then $\operatorname{Det}(S(X ; \lambda, I))=\operatorname{Det}\left(S_{1}(X ; \lambda, I)\right)=\frac{y^{2}}{I r^{2}} \neq 0$. But this is not possible, and thus $\lambda r^{3} \neq 1$. Since $S_{1}(X ; \lambda, I)$ and $S(X ; \lambda, I)$ have identical row and 
null spaces, $S_{1}(X ; \lambda, I)$ is singular, but not the zero matrix. Now its upper right entry is nonzero, and so its top row spans its row space. Hence

$$
\left[\begin{array}{l}
-\frac{x}{y}\left(\frac{1-\lambda r^{3}}{3}\right) \\
\frac{\lambda r^{3}}{I}-\frac{1-\lambda r^{3}}{3}
\end{array}\right]
$$

is a basis for its null space. Thus $K(X ; \lambda, I)$ is finite and given by

$$
K(X ; \lambda, I)=\frac{\left(\frac{\lambda r^{3}}{I}-\frac{1-\lambda r^{3}}{3}\right)}{-\frac{x}{y}\left(\frac{1-\lambda r^{3}}{3}\right)}=\frac{y}{x}\left[\frac{3+I}{I}\right]\left[\frac{\left(\frac{I}{3+I}\right)-\lambda r^{3}}{1-\lambda r^{3}}\right] .
$$

ii) Assume $X=\left[\begin{array}{l}x \\ y\end{array}\right]$ is on the singularity locus and $x=0$. From Lemma $[5.1$ $x=0$ implies $N(X ; \lambda, I) \neq R^{2}$. Now the bottom row of $S(X ; \lambda, I)$ satisfies $\left[\frac{x y}{r^{2}}-\right.$ $\left.\frac{\lambda r^{3}}{I} y \quad \frac{y^{2}}{r^{2}}-\frac{1-\lambda r^{3}}{3}\right]=\left[\begin{array}{ll}-\frac{\lambda r^{3}}{I} y & \frac{2+\lambda r^{3}}{3}\end{array}\right]$. Since the row on the right is not the zero row, it spans the row space of $S(X ; \lambda, I)$. Therefore $K(X ; \lambda, I)=\frac{\left(\frac{\lambda r^{3}}{L} y\right)}{\left(\frac{2+\lambda r^{3}}{2}\right)}=\frac{3 \lambda r^{3} y}{I\left(2+\lambda r^{3}\right)}=$ $\frac{y}{2+1}$. The complete result follows by using Part ii) of Lemma 5.2 .

iii) From Lemmas 5.1 and 5.2 it is seen that $X=\left[\begin{array}{c}-\sqrt[3]{\frac{1}{\lambda}} \\ 0\end{array}\right]$ is on the singularity locus and $N(X ; \lambda, I) \neq R^{2}$. For this $X$, the top row of $S(X ; \lambda, I)$ is nonzero and equal to $\left[1+\frac{\left(1+\sqrt[3]{\frac{1}{\lambda}}\right)}{I} \quad 0\right]$. Thus $N(X ; \lambda, I)$ is spanned by $\left[\begin{array}{l}0 \\ 1\end{array}\right]$.

iv) From Lemmas 5.1 and 5.2 , with $\lambda \neq \frac{1}{(I+1)^{3}}$, it is seen that $X=\left[\sqrt[3]{\frac{1}{\lambda}}\right]$ is on the singularity locus and $N(X ; \lambda, I) \neq R^{2}$. For this $X$, the top row of $S(X ; \lambda, I)$ is equal to $\left[\frac{\left(I+1-\sqrt[3]{\frac{1}{\lambda}}\right)}{I} 0\right]$ and is nonzero. Thus $N(X ; \lambda, I)$ is spanned by $\left[\begin{array}{l}0 \\ 1\end{array}\right]$.

v) From Lemmas 5.1 and [5.2, with $\lambda \neq \frac{1}{(I+1)^{3}}$, it is seen that $X=\left[\begin{array}{c}\eta(\lambda, I) \\ 0\end{array}\right]$ is on the singularity locus and $N(X ; \lambda, I) \neq R^{2}$. Also, Lemma [5.2, Part i) gives $\eta(\lambda, I) \neq \sqrt[3]{\frac{1}{\lambda}}$ when $\lambda \neq \frac{1}{(I+1)^{3}}$. So for the given $X$ the bottom row of $S(X ; \lambda, I)$ is equal to $\left[0-\left(\frac{1-\lambda(\eta(\lambda, I))^{3}}{3}\right)\right]$ and is nonzero. Thus $N(X ; \lambda, I)$ is spanned by $\left[\begin{array}{l}1 \\ 0\end{array}\right]$ and $K(X ; \lambda, I)=0$.

According to Proposition 4.1 were one-point isolation not to hold when $G\left(X_{n}\right)$ is in zero-one form, then the points $X_{1}, X_{2}, \ldots, X_{n-1}$ would all be located on the singularity locus. Additionally, there would exist a unit vector $T$ which would be contained in $\bigcap_{j=1}^{n-1} N\left(X_{j} ; \lambda, I\right)$.

Our goal is to show this is impossible when $n>4$. Thus our next step is to determine an upper bound on the number of points on the singularity locus for which a given unit vector $T$ can be contained in each of the corresponding null spaces. We will divide the task into cases. We begin with horizonal and vertical unit vectors.

Proposition 5.4. Given any $\lambda, I>0$,

i) if $T=\left[\begin{array}{l}0 \\ 1\end{array}\right]$, there are exactly two $X$ on the singularity locus for which $T \in$ $N(X ; \lambda, I)$,

ii) if $T=\left[\begin{array}{l}1 \\ 0\end{array}\right]$, there are at most three $X$ on the singularity locus for which $T \in N(X ; \lambda, I)$.

Proof. i) If $\lambda \neq \frac{1}{(I+1)^{3}}$, Lemma 5.1 implies that $N(X ; \lambda, I)$ is one dimensional for all $X$ on the singularity locus. In this case, Proposition 5.3 gives the slope of the line that constitutes $N(X ; \lambda, I)$. The result follows from the fact that $K(X ; \lambda, I)$ is 
undefined if and only if $X=\left[ \pm \sqrt[3]{\frac{1}{\lambda}}\right]$. Again using Lemma 5.1 and Proposition 5.3 if $\lambda=\frac{1}{(I+1)^{3}}$, it is seen that $N(X ; \lambda, I)$ is two dimensional when $X=\left[\sqrt[3]{\frac{1}{\lambda}}\right]$ and, in the one dimensional cases, $K(X ; \lambda, I)$ is undefined if and only if $X=\left[-\sqrt[3]{\frac{1}{\lambda}}\right]$. So in any case, for any $\lambda, I>0, T \in N(X ; \lambda, I)$, if and only if $X=\left[ \pm \sqrt[3]{\frac{1}{\lambda}}\right]$.

ii) First, let $X$ be on the $x$ axis. From Lemma [5.2, if $\lambda \neq \frac{1}{(I+1)^{3}}$, there are three such $X$. According to Proposition 5.3, the only one of the these for which $K(X ; \lambda, I)=0$, is $X=\left[\begin{array}{c}\eta(\lambda, I) \\ 0\end{array}\right]$. If $\lambda=\frac{1}{(I+1)^{3}}$, there ae two such $X, X=\left[ \pm \sqrt[3]{\frac{1}{\lambda}}\right]$. $N(X ; \lambda, I)$ is two dimensional when $X=\left[\sqrt[3]{\frac{1}{\lambda}}\right]$ and $K(X ; \lambda, I)$ is undefined when $X=\left[-\sqrt[3]{\frac{1}{\lambda}}\right]$. Thus, in any case, there is exactly one point on the $x$ axis for which $T \in N(X ; \lambda, I)$. Now, if $X$ is not on the $x$ axis, Proposition 5.3 reveals that $K(X ; \lambda, I)=0$ if and only if $x \neq 0$ and $\lambda r^{3}=\left(\frac{I}{3+I}\right)$. Substituting $r=\sqrt[3]{\left(\frac{I}{3+I}\right) \frac{1}{\lambda}}$ into the equation of the singularity locus (as given in Proposition 5.2) and solving for $\cos (\phi)$ gives $\cos (\phi)=0$ and $\cos (\phi)=\left(\frac{1}{3+I}\right) \sqrt[3]{\left(\frac{I}{3+I}\right) \frac{1}{\lambda}}$. Since $x \neq 0$, the solution $\cos (\phi)=0$ does not corresponding to a point on the singularity locus at which $K(X ; \lambda, I)=0$. The solution $\cos (\phi)=\left(\frac{1}{3+I}\right) \sqrt[3]{\left(\frac{I}{3+I}\right) \frac{1}{\lambda}}$ corresponds to at most two points on it (one in the first and one in the fourth quadrant) where $K(X ; \lambda, I)=0$. However, it may not correspond to any points if $\left(\frac{1}{3+I}\right) \sqrt[3]{\left(\frac{I}{3+I}\right) \frac{1}{\lambda}}>1$. So in total there are at most three points on the singularity locus for which $T \in N(X ; \lambda, I)$.

Determining the upper bound in the cases in which $T$ is neither vertical nor horizontal requires greater effort. A new parameter $k$ will be used. It represents the slope of $T$, defined to equal $\frac{t_{2}}{t_{1}}$ when $T=\left[\begin{array}{c}t_{1} \\ t_{2}\end{array}\right]$ and $t_{1} \neq 0$. In the subsequent analysis $k$ will be taken to be positive. The results for $k<0$ will follow from those established for $k>0$.

The upper bound we seek equals the maximum number of points common to two polar loci. The polar equations used in defining the loci are

Polar Equation 1 (PE1): $r=\sqrt[3]{\frac{I}{\lambda(3+I)}}(\Omega(\phi))^{1 / 3}$, where

$$
\Omega(\phi)=\frac{\sin (\phi)-k \cos (\phi)}{\sin (\phi)-\left(\frac{k I}{3+I}\right) \cos (\phi)} .
$$

Polar Equation $2(\mathrm{PE} 2): r=\Theta(\phi)$, where

$$
\Theta(\phi)=\frac{\left(3+I-I k^{2}\right) \cos (\phi) \sin (\phi)+k(3+2 I) \sin ^{2}(\phi)-k(I+1)}{\sin (\phi)-k \cos (\phi)} .
$$

PE1 is obtained by using the formula for $K(X ; \lambda, I)$ that appears in Proposition 5.3 Part i). $K(X ; \lambda, I)$ is replaced by $k, y$ by $r \sin (\phi)$, and $x$ by $r \cos (\phi)$, and then the equation is solved for $r$, where it is assumed that $\sin (\phi) \neq 0$ and $\cos (\phi) \neq 0$.

PE2 is obtained by using the formula for the singularity locus as given in Proposition 5.2 together with PE1. (The latter yields $\lambda r^{3}=\frac{I}{(3+I)} \Omega(\phi)$.) Making the following replacements in the singularity locus formula and then solving for $r$ results in PE2: $\lambda^{2} r^{7}$ by $r\left(\frac{I}{(3+I)} \Omega(\phi)\right)^{2}, \lambda^{2} r^{6}$ by $\left(\frac{I}{(3+I)} \Omega(\phi)\right)^{2}, \lambda r^{4}$ by $r\left(\frac{I}{(3+I)} \Omega(\phi)\right)$, and $\lambda r^{3}$ by $\frac{I}{(3+I)} \Omega(\phi)$. 
Thus, if $X=\left[\begin{array}{l}x \\ y\end{array}\right]$ is on the singularity locus, where $x \neq 0, y \neq 0$, and $T \in$ $N(X ; \lambda, I)$ is a unit vector with slope $k$, then $X$ satisfies PE1 and PE2, where $r=\sqrt{x^{2}+y^{2}}$ is positive in each of the equations. The two polar loci (Locus A and Locus (B), for which we seek an upper bound on the number of points in common, are given by PE1 and PE2 over the intervals on which both yield a positive value of $r$. The next lemma gives the intervals. The loci are defined after the lemma. The following angles will be used:

$$
\begin{aligned}
& \phi_{1}=\arctan \left(\frac{-3-I+I k^{2}+\sqrt{8 k^{2}+\left(3+I+I k^{2}\right)^{2}}}{2 k(2+I)}\right), \\
& \phi_{2}=\arctan \left(\frac{-3-I+I k^{2}-\sqrt{8 k^{2}+\left(3+I+I k^{2}\right)^{2}}}{2 k(2+I)}\right)+\pi, \\
& \phi_{3}=\arctan \left(\frac{-3-I+I k^{2}+\sqrt{8 k^{2}+\left(3+I+I k^{2}\right)^{2}}}{2 k(2+I)}\right)+\pi, \\
& \phi_{4}=\arctan \left(\frac{-3-I+I k^{2}-\sqrt{8 k^{2}+\left(3+I+I k^{2}\right)^{2}}}{2 k(2+I)}\right)+2 \pi .
\end{aligned}
$$

Lemma 5.3. i) For $0 \leq \phi<2 \pi$ and $k>0, \Omega(\phi)$ is defined and positive if and only if one of the following conditions hold: $0 \leq \phi<\arctan \left(\frac{k I}{3+I}\right) ; \arctan (k)<\phi<$ $\arctan \left(\frac{k I}{3+I}\right)+\pi ; \arctan (k)+\pi<\phi<2 \pi$.

ii) For $0 \leq \phi<2 \pi$ and $k>0, \Theta(\phi)$ is defined and positive if and only if one of the following conditions hold: $0 \leq \phi<\phi_{1} ; \arctan (k)<\phi<\phi_{2} ; \phi_{3}<\phi<\arctan (k)+\pi$; $\phi_{4}<\phi<2 \pi$.

iii) For $0 \leq \phi<2 \pi$ and $k>0, \Omega(\phi)$ and $\Theta(\phi)$ are both defined and positive if and only if one of the following conditions hold: $0 \leq \phi<\arctan \left(\frac{k I}{3+I}\right) ; \arctan (k)<$ $\phi<\phi_{2} ; \phi_{4}<\phi<2 \pi$.

Proof. i) The sign changes of $\Omega(\phi)$ occur at points where either the numerator or denominator is 0 . These are determined by either $\tan (\phi)=k$ or $\tan (\phi)=\frac{k I}{3+I}$. An examination of the sign of $\Omega(\phi)$ between successive points at which there is a sign change gives the result.

ii) The sign changes of $\Theta(\phi)$ occur at points where either the numerator or denominator is 0 . The denominator is 0 when $\phi=\arctan (k)$ or $\phi=\arctan (k)+$ $\pi$. Since the numerator is nonzero when $\cos (\phi)=0$, we can find its zeros by dividing through by $\cos ^{2}(\phi)$ and setting the result to 0 . This equation can be written as $\beta(\tan (\phi))=0$, where $\beta$ is a polynomial which given as a function of $t$ is $\beta(t)=k(2+I) t^{2}+\left(3+I-I k^{2}\right) t-k(1+I)$. The solutions are $\tan (\phi)=$ $\frac{-3-I+I k^{2} \pm \sqrt{8 k^{2}+\left(3+I+I k^{2}\right)^{2}}}{2(2+I) k}$. Since the plus sign yields a positive value for $\tan (\phi)$ and the minus sign a negative value for $\tan (\phi)$, the solutions in terms of $\phi, 0 \leq \phi<$ $2 \pi$, are, in increasing order of magnitude, $\phi_{1}, \phi_{2}, \phi_{3}, \phi_{4}$.

To insert the denominator zeros in this sequence, we use the fact that $k>$ $\frac{-3-I+I k^{2}+\sqrt{8 k^{2}+\left(3+I+I k^{2}\right)^{2}}}{2 k(2+I)}$. This is seen from the relations

$$
\begin{aligned}
\left(2 k^{2}(2+I)\right. & \left.-\left(-3-I+I k^{2}\right)\right)^{2}=\left(\left(3+I+I k^{2}\right)+4 k^{2}\right)^{2} \\
& =\left(3+I+I k^{2}\right)^{2}+8 k^{2}\left(I k^{2}+3+I\right)+16 k^{4}>\left(3+I+I k^{2}\right)+8 k^{2} .
\end{aligned}
$$




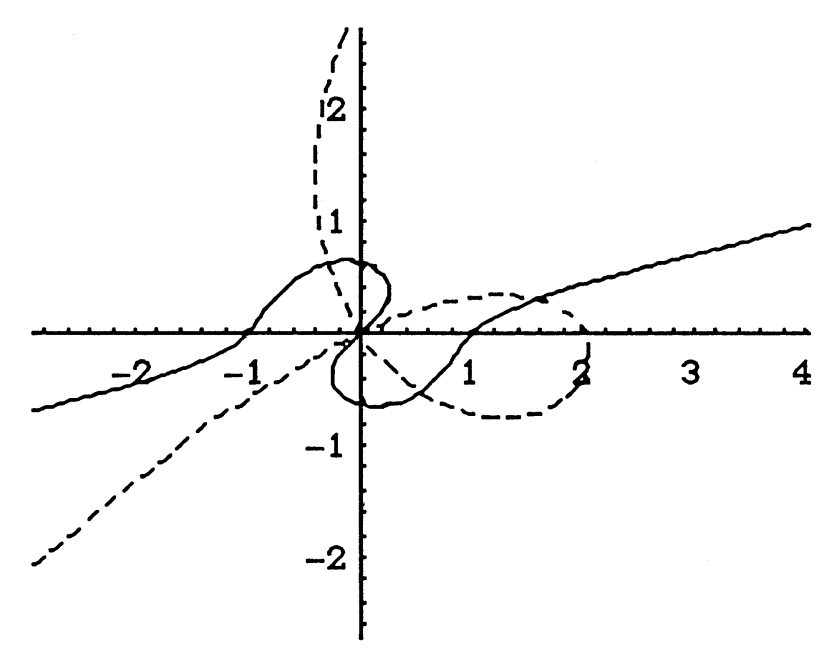

Figure 5.3. PE1 (solid curve), PE2 (dashed curve); $\lambda=1, I=$ $1, k=1$

Thus $0<\phi_{1}<\arctan (k)<\phi_{2}<\phi_{3}<\arctan (k)+\pi<\phi_{4}<2 \pi$. Since the numerator and denominator zeros are all simple there is a sign change of $\Theta(\phi)$ at each zero. Combining this with $\Theta(0)=I+1>0$ yields the result.

iii) We use the fact that $\frac{k I}{3+I}<\frac{-3-I+I k^{2}+\sqrt{8 k^{2}+\left(3+I+I k^{2}\right)^{2}}}{2 k(2+I)}$, which is seen from the relations $\frac{2 k^{2} I(2+I)}{3+I}-\left(-3-I+I k^{2}\right)=\left(3+I+\left(\frac{I+1}{3+I}\right) I k^{2}\right)<3+I+I k^{2}<$ $\sqrt{8 k^{2}+\left(3+I+I k^{2}\right)^{2}}$. From this it follows that $\arctan \left(\frac{k I}{3+I}\right)<\phi_{1}$ and $\arctan \left(\frac{k I}{3+I}\right)$ $+\pi<\phi_{3}$. Hence $0<\arctan \left(\frac{k I}{3+I}\right)<\phi_{1}<\arctan (k)<\phi_{2}<\arctan \left(\frac{k I}{3+I}\right)+\pi<$ $\phi_{3}<\arctan (k)+\pi<\phi_{4}<2 \pi$, and the intervals on which both functions are defined and positive follow from those in parts i, ii.

In light of Part iii of the previous lemma we define the loci as the points in the plane determined by the following relations:

Locus A. $r=\sqrt[3]{\frac{I}{\lambda(3+I)}}(\Omega(\phi))^{1 / 3} ; \arctan (k)<\phi<\phi_{2}$ or $\phi_{4}<\phi<\arctan \left(\frac{k I}{3+I}\right)$ $+2 \pi$.

Locus B. $r=\Theta(\phi) ; \arctan (k)<\phi<\phi_{2}$ or $\phi_{4}<\phi<\arctan \left(\frac{k I}{3+I}\right)+2 \pi$.

For $\lambda=1, I=1, k=1$, Figure 5.3 illustrates the loci by presenting plots of PE1 and PE2 over the $\phi$ intervals, respectively given in parts $\mathrm{i}$, ii of the previous lemma, for which the $r$ values are positive. The plots necessarily have been truncated for $\phi$ near the singular values. The angles in degrees are approximately $\arctan (k)=45^{\circ}$, $\arctan \left(\frac{k I}{3+I}\right)=14.04^{\circ}, \phi_{1}=24.58^{\circ}, \phi_{2}=124.46^{\circ}, \phi_{3}=204.58^{\circ}, \phi_{4}=304.46^{\circ}$.

Note that Locus $[\mathrm{A}$ is a subset of of the solid curve and Locus [B] a subset of the dashed curve. Note also that $X=0$ is not common to the two loci.

The number of intersections of the two loci on $\arctan (k)<\phi<\phi_{2}$ will be found by showing that $r$ as defined in Locus $\mathrm{A}$ is increasing, and $r$ as defined in Locus $\mathrm{B}$ is decreasing.

Lemma 5.4. For any $k, I>0, \Omega^{\prime}(\phi)>0$ on the interval $\arctan (k)<\phi<\phi_{2}$. 
Proof. The result follows from the fact that $\Omega^{\prime}(\phi)=\frac{3 k}{(3+I)\left(\sin (\phi)-\frac{I k}{3+I} \cos (\phi)\right)^{2}}>0$.

Lemma 5.5. For any $k, I>0, \Theta^{\prime}(\phi)<0$ on the interval $\arctan (k)<\phi<\phi_{2}$.

Proof. In the formula for $\Theta(\phi)$, replace $\sin ^{2}(\phi)$ by $\frac{1-\cos (2 \phi)}{2}$ and $\sin (\phi) \cos (\phi)$ by $\frac{\sin (2 \phi)}{2}$. Differentiate by the quotient rule and then divide the numerator of the derivative by $\cos (\phi) \cos (2 \phi)$. Express this quotient in terms of $\tan (\phi)$ and multiply the result by $\cos (\phi) \cos (2 \phi)$ where $\cos (2 \phi)$ is expressed in terms of $\tan (\phi)$. Combine expressions and obtain $\Theta^{\prime}(\phi)=\frac{\sec (\phi)}{(k-\tan (\phi))^{2}\left(1+\tan ^{2}(\phi)\right)} Q(\tan (\phi))$, where $Q$ is a polynomial, which when given as a function of $t$ is

$$
Q(t)=k\left(k^{2} I-2\right)-k^{2}(5+3 I) t+k(4+3 I) t^{2}-\left(3+I+2 k^{2}\right) t^{3} .
$$

Since the zeros of $Q^{\prime}(t)$ are $\frac{(4+3 I) k \pm \sqrt{-k^{2}\left(29+18 I+30 k^{2}+18 I k^{2}\right)}}{3\left(3+I+2 k^{2}\right)}$ and are both nonreal, it follows that $Q$ is a decreasing function of $t$ for all $t$. From $Q(k)=-2 k\left(k^{2}+1\right)^{2}<0$ it then follows that $\Theta^{\prime}(\phi)<0$ for $\arctan (k)<\phi<\frac{\pi}{2}$. The singularity at $\frac{\pi}{2}$ in the above representation of $\Theta^{\prime}(\phi)$ is removable and $\Theta^{\prime}\left(\frac{\pi}{2}\right)=-\left(3+I+2 k^{2}\right)$. To show $\Theta^{\prime}(\phi)<0$ when $\frac{\pi}{2}<\phi<\phi_{2}$ we need to show $Q(t)>0$ for $-\infty<$ $t<\frac{-3-I+I k^{2}-\sqrt{8 k^{2}+\left(3+I+I k^{2}\right)^{2}}}{2 k(2+I)}$. Since $Q$ is positive for large negative $t$ and is decreasing, we need only show $Q\left(\frac{-3-I+I k^{2}-\sqrt{8 k^{2}+\left(3+I+I k^{2}\right)^{2}}}{2 k(2+I)}\right)>0$. This will be true if $Q\left(\frac{-3-I+I k^{2}-\left(3+I+I k^{2}\right)}{2 k(2+I)}\right)=Q\left(\frac{-3-I}{k(2+I)}\right)>0$. Since in the evaluation of $Q$ at $t=\frac{-3-I}{k(2+I)}$ the cubic and quadratic terms are positive, it need only be shown that $k\left(k^{2} I-2\right)-k^{2}(5+3 I)\left(\frac{-3-I}{k(2+I)}\right)>0$. But this is true because the product of the left side of the inequality and $\frac{2+I}{k}$ is equal to $(2+I) I k^{2}+11+12 I+3 I^{2}$.

The number of intersections on $\phi_{4}<\phi<\arctan \left(\frac{k I}{3+I}\right)+2 \pi$ will be shown to be bounded above by two. This will be accomplished by showing that $r$ as defined in Locus $\mathrm{A}$ has a positive second derivative and $r$ as defined in Locus $\mathrm{B}$ has a negative second derivative.

Lemma 5.6. For any $k, I>0$, the function $r(\phi)=(\Omega(\phi))^{1 / 3}$ has a positive second derivative on the interval $\phi_{4}<\phi<\arctan \left(\frac{k I}{I+3}\right)+2 \pi$.

Proof. According to part iii) of Lemma 5.3. $\Omega(\phi)$ is defined and positive on this interval. We can express the second derivative of $r$ as $r^{\prime \prime}(\phi)=\frac{\Omega(\phi) \Omega^{\prime \prime}(\phi)-\frac{2}{3}\left(\Omega^{\prime}(\phi)\right)^{2}}{3(\Omega(\phi))^{5 / 3}}$. By performing some extensive manipulation, after differentiating with $\Omega(\phi)$ in the form $\Omega(\phi)=\frac{\tan (\phi)-k}{\tan (\phi)-\left(\frac{k I}{3+1}\right)}$, it can be shown that $\Omega(\phi) \Omega^{\prime \prime}(\phi)-\frac{2}{3}\left(\Omega^{\prime}(\phi)\right)^{2}=$ $\frac{6 k(3+I)^{2} R(\tan (\phi))}{((3+I) \tan (\phi)-I k)^{4}}$ where $R$ is a polynomial, which when given as a function of $t$ is $R(t)=\left(1+t^{2}\right)\left(-k(1+I) t^{2}+\left(-3-I+I k^{2}\right) t+(2+I) k\right)$. The second factor in $R(t)$ is positive between its real zeros which are $\frac{-3-I+I k^{2} \pm \sqrt{8 k^{2}+\left(3+I+I k^{2}\right)^{2}}}{2 k(1+I)}$. But $\frac{-3-I+I k^{2}-\sqrt{8 k^{2}+\left(3+I+I k^{2}\right)^{2}}}{2 k(1+I)}=\left(\frac{2+I}{1+I}\right) \tan \left(\phi_{4}\right)<\tan \left(\phi_{4}\right)$ and

$$
\frac{-3-I+I k^{2}+\sqrt{8 k^{2}+\left(3+I+I k^{2}\right)^{2}}}{2 k(1+I)}=\left(\frac{2+I}{1+I}\right) \tan \left(\phi_{1}\right)>\tan \left(\phi_{1}\right)>\frac{k I}{3+I} .
$$

Hence $R(\tan (\phi))$ is positive in the interval $\phi_{4}<\phi<\arctan \left(\frac{k I}{I+3}\right)+2 \pi$ and the result follows. 
Lemma 5.7. For any $k, I>0, \Theta(\phi)$ has a negative second derivative on the interval $\phi_{4}<\phi<\arctan \left(\frac{k I}{I+3}\right)+2 \pi$.

Proof. Extensive manipulation reveals that $\Theta^{\prime \prime}(\phi)=\frac{\cos (\phi) w(\tan (\phi))}{(k-\tan (\phi))^{3}} . \quad w(t)=$ $(t-k)^{2} \beta(t)-4\left(k+k^{3}\right)\left(t^{2}+1\right)^{2}$ and $\beta(t)$ is the polynomial defined in Part ii of the proof of Lemma 5.3. From the proofs of Parts ii, iii of this same lemma it is seen that the two distinct real roots of $\beta(t)=0$ are $t=\tan \left(\phi_{1}\right)$ and $t=\tan \left(\phi_{4}\right)$ and that $\beta(t)$ is negative when $t$ is between these roots. Further, $\frac{3 \pi}{2}<\phi_{4}<\arctan \left(\frac{k I}{I+3}\right)+2 \pi<$ $\phi_{1}+2 \pi<\arctan (k)+2 \pi<2 \pi+\frac{\pi}{2}$. Thus, if $\phi_{4}<\phi<\arctan \left(\frac{k I}{I+3}\right)+2 \pi$, then $\tan \left(\phi_{4}\right)<\tan (\phi)<\tan \left(\phi_{1}\right), k-\tan (\phi)>0, \cos (\phi)>0$, and $\beta(\tan (\phi))<0$. Hence $w(\tan (\phi))<0$ and therefore $\Theta^{\prime \prime}(\phi)<0$.

Proposition 5.5. For any $k, I, \lambda>0$, Locus $A$ and Locus $B$ have at most three points in common.

Proof. Lemmas 5.4 and 5.5 imply that $h(\phi)=\sqrt[3]{\frac{I}{\lambda(3+I)}}(\Omega(\phi))^{1 / 3}-\Theta(\phi)$ is a strictly increasing function of $\phi$ on the interval $\arctan (k)<\phi<\phi_{2}$. Since $\Omega(\arctan (k))=0$, $\Theta(\phi) \rightarrow+\infty$ as $\phi \rightarrow(\arctan (k))^{+}, \Omega\left(\phi_{2}\right)>0$, and $\Theta\left(\phi_{2}\right)=0, h$ takes on both positive and negative values on this interval. Hence the loci have exactly one point in common when $\phi$ is in the interval $\arctan (k)<\phi<\phi_{2}$.

Lemmas 5.6 and 5.7imply that $h^{\prime \prime}(\phi)>0$ on the interval $\phi_{4}<\phi<\arctan \left(\frac{k I}{I+3}\right)+$ $2 \pi$. Hence $h$ can have at most two distinct zeros on this interval. Thus the loci have at most three points in common.

Proposition 5.6. For any $\lambda, I>0$ and any unit vector $T$, there are at most three $X$ on the singularity locus for which $T \in N(X ; \lambda, I)$.

Proof. The cases in which $T$ is horizontal or vertical are covered by Proposition 5.4 When $T$ is a nonvertical unit vector with slope $k \neq 0$ we break the proof into cases:

Case a): $k>0$.

Let $X=\left[\begin{array}{l}x \\ y\end{array}\right]$ and assume $T \in N(X ; \lambda, I)$.

Subcase i) $x \neq 0, y \neq 0$ : According to the definition of the loci, $X$ is on both Locus A and Locus B.

Subcase ii) $x=0$ : Since $k>0$, according to Lemma 5.2 Part ii and Proposition 5.3 Part ii, $X=\left[+\sqrt[3]{\frac{1}{\lambda\left(\frac{I}{3+I}\right)}}\right]$ and $k=\frac{+\sqrt[3]{\frac{1}{\lambda}\left(\frac{I}{3+I}\right)}}{2+I}$. Taking $\phi=\frac{\pi}{2}$ and $r=+\sqrt[3]{\frac{1}{\lambda}\left(\frac{I}{3+I}\right)}$, it is seen that $X$ is on both Locus $\mathrm{A}$ and Locus B

Subcase iii) $y=0$ : It follows from Lemma 5.1 Lemma 5.2 Part i, and Proposition [5.3 Parts iii, iv, $\mathrm{v}$ that $\lambda=\frac{1}{(1+I)^{3}}$ and $X=\left[\begin{array}{c}I+1 \\ 0\end{array}\right]=\left[\sqrt[3]{\frac{1}{\lambda}} 0\right]$. In this case, the polar coordinates, $(r, \phi)$, of $X$ are $\left(\sqrt[3]{\frac{I}{\lambda}}, 0\right)$. Since $\sqrt[3]{\frac{1}{\lambda}}=\sqrt[3]{\frac{I}{\lambda(3+I)}}(\Omega(0))^{1 / 3}, X$ is on Locus A Also, since $\sqrt[3]{\frac{1}{\lambda}}=I+1=\Theta(0), X$ is on Locus B

So whenever $k>0$ and $T \in N(X ; \lambda, I), X$ is common to both Locus $\mathrm{A}$ and Locus B The result follows from Proposition 5.5 which states that at most three such $X$ exist.

Case b) $k<0$ : Let $X=\left[\begin{array}{l}x \\ y\end{array}\right]$ and assume $T \in N(X ; \lambda, I)$. Set $X^{*}=\left[\begin{array}{c}x \\ -y\end{array}\right]$ and let $T^{*}$ be a unit vector with slope $-k>0$. According to Proposition [5.2 $X^{*}$ is on the singularity locus. From Lemmas 5.1, 5.2 and Proposition 5.3 it follows that $T^{*} \in N\left(X^{*} ; \lambda, I\right)$. From Case a) there are at most three such $X^{*}$ and hence for $k<0$ there are at most three $X$ for which $T \in N(X ; \lambda, I)$. 
The notation of Definition 4.1 is used in the following corollary.

Corollary 5.1. If $X_{n 0}=0$ and $\left\{X_{10}, X_{20}, \ldots, X_{n 0} ; m_{1}, m_{2}, \ldots, m_{n}\right\}$ is a zero-one form planar central configuration, then for any unit vector $T \in R^{2}$, there exists at most three distinct values of $j, 1 \leq j<n$, for which $\operatorname{Lim}_{h \rightarrow 0^{+}} \frac{\left|P_{j}\left(X^{0}+h T_{n}^{0}\right)\right|}{h}=0$.

Proof. If the limit is $0, D P_{j}\left(X^{0}\right) T_{n}^{0}=0$. In the "non-Definition 4.1 notation" this means $P_{j}^{\prime}\left(X_{n 0}\right) T=0$. Hence $X_{j 0}$ is on the singularity locus and $T \in N\left(X_{j 0} ; \lambda, I\right)$ and the result follows from Proposition 5.6

Proposition 5.7 (One-Point Isolation; Zero-One Form). If $n>4$ and $G\left(X_{n}\right)$ is a zero-one planar central configuration, there is a neighborhood of $X_{n}=0$, such that if $Z \neq X_{n}$ is in the neighborhood, then $G(Z)$ is not central.

Proof. If such a neighborhood did not exist there would exist a sequence of planar central configurations $\left\{G\left(Z_{k}\right)\right\}$ for which $Z_{k} \neq X_{n}$ and $Z_{k} \rightarrow X_{n}=0$. Hence, according to Proposition 4.1 and the definitions associated with the singularity locus, there would exist a unit vector $T$ for which $T \in N\left(X_{j} ; \lambda, I\right), j=1,2, \ldots, n-1$. $I>0$ is the moment of inertia of $G\left(X_{n}\right)$ about the center of mass $(1,0)$, and $\lambda=\frac{U}{I}>0$, where $U$ is the Newtonian potential of $G\left(X_{n}\right)$. But, since $n-1>3$, the existence of $n-1$ distinct $X_{j}$ for which $T \in N\left(X_{j} ; \lambda, I\right)$ would contradict Proposition 5.6. Hence, such a neighborhood exists, and the proposition is proved.

\section{The MAIN RESUlts}

In this section we prove the general one-point isolation theorem and then use it to prove that only a finite number of planar central configurations can be generated by augmenting a given $n-1$ point planar configuration with an additional mass. We also gather results used in these proofs to state general results about directional derivatives and derivative matrices at central configurations. Finally, we use these to describe how much destruction of the centrality property must occur when a single point in a central configuration is perturbed.

Theorem 6.1 (One-Point Isolation). If $n$ is greater than 2 and not equal to 4 , and if $G\left(X_{n}\right)$ is a planar central configuration of $n$ points, then there is a neighborhood of $X_{n}$, such that if $Z \neq X_{n}$ is in the neighborhood, then $G(Z)$ is not a planar central configuration.

Proof. If $n=3$, the result follows from the fact that there are exactly $5 Z$ for which $G(Z)$ is a central configuration.

In light of Theorem 4.2, when $n$ is greater than 4 , it is only necessary to consider the case in which the distinguished point $X_{n}$ is not the center of mass. In this case, if such a neighborhood did not exist, then, because the central configuration property is maintained by translation, rotation, or change of scale, there would exist a zero-one planar central configuration of more than 4 points for which onepoint isolation did not hold. Since this would contradict Proposition 5.7, such a neighborhood exists and the theorem is proved.

It should be noted that the finite number stated in the next theorem may in fact be zero. Examples in which this occurs can be created by tightly clustering proper subsets of the $n-1$ points. See Buck [1].

Theorem 6.2. Let $n$ be greater than 2 and not equal to 4 , and let $X_{1}, X_{2}, \ldots, X_{n-1}$ be distinct fixed points in a plane at which are located nonzero masses $m_{1}, m_{2}$, 
$\ldots, m_{n-1}$. Then, given an $n$th mass $m_{n}>0$, there are only a finite number of $Z$ in the same plane, distinct from $X_{1}, X_{2}, \ldots, X_{n-1}$, at which one may locate this additional mass and thereby make the $n$-point configuration $G(Z)=\left\{X_{1}, X_{2}, \ldots, X_{n-1}\right.$, $\left.Z ; m_{1}, m_{2}, \ldots, m_{n-1}, m_{n}\right\}$ central.

Proof. Assume there were an infinite number of $Z$ in the same plane which would make $G(Z)$ a planar central configuration. It follows that there would then exist a sequence $Y_{1}, Y_{2}, Y_{3}, \ldots$ of distinct points in this plane which had the property that the corresponding planar configurations $G\left(Y_{i}\right)$ were central and which also satisfied at least one of the following three conditions: i) the sequence is bounded and contains a subsequence which converges to an $X_{n}$ that is distinct from $X_{1}, X_{2}, \ldots, X_{n-1}$, ii) the sequence is bounded and contains a subsequence which converges to one of the $X_{j}, j=1,2, \ldots, n-1$, iii) the sequence is unbounded. The proof consists of considering the three cases corresponding to conditions i, ii, and iii, and showing that in each case such a sequence $Y_{1}, Y_{2}, Y_{3}, \ldots$ cannot exist.

Case i): Let $Z_{1}, Z_{2}, Z_{3}, \ldots$ be a subsequence with the property that $Z_{k} \rightarrow X_{n}$ and $G\left(Z_{k}\right)$ is central. Using the notation of Section $2, G(Z)$ is central when $A(Z)-$ $\lambda(Z) Q(Z)=0$ and $P_{j}(Z)=0, j=1,2, \ldots, n-1$. Since the expressions on the left side of these equalities are continuous functions of $Z$ at $Z=X_{n}$, it follows that $G\left(X_{n}\right)$ is central. But then $Z_{k} \rightarrow X_{n}$ and $G\left(Z_{k}\right)$ central contradicts Theorem 6.1 Hence condition i in conjunction with the centrality of the configurations $G\left(Y_{i}\right)$ is impossible.

Case ii): Let $Z_{1}, Z_{2}, Z_{3}, \ldots$ be a subsequence with the property that $Z_{k} \rightarrow X_{j}$ and $G\left(Z_{k}\right)$ is central, where $j$ is some fixed integer between 1 and $n-1$. Since $r_{k}=\left|Z_{k}-X_{j}\right| \rightarrow 0$, it follows that $\left|A_{j}\left(Z_{k}\right)\right| \sim \frac{m_{n}}{r_{k}^{2}}, U\left(Z_{k}\right) \sim \frac{m_{n} m_{j}}{r_{k}}$, and $I\left(Z_{k}\right) \rightarrow$ $I^{*} \neq 0$ (since $n>2$ ). Further, $\left|Q_{j}\left(Z_{k}\right)\right| \rightarrow C^{*}<\infty$, and thus for large $k$, $\left|\lambda\left(Z_{k}\right) Q_{j}\left(Z_{k}\right)\right|<\frac{2\left(C^{*}+1\right)\left(m_{n} m_{j}+1\right)}{I^{*} r_{k}}<\frac{m_{n}}{2 r_{k}^{2}}<\left|A_{j}\left(Z_{k}\right)\right|$. This contradicts the fact that $P_{j}\left(Z_{k}\right)=0$ for all $k$. Hence condition ii in conjunction with the centrality of the configurations $G\left(Y_{i}\right)$ is impossible.

Case iii) Let $Z_{1}, Z_{2}, Z_{3}, \ldots$ be a subsequence with the property that $\left|Z_{k}\right| \rightarrow \infty$ and $G\left(Z_{k}\right)$ is central. With $M=\sum_{j=1}^{n-1} m_{j}$ and $r_{k}=\left|Z_{k}\right|$, we have $I\left(Z_{k}\right) \sim$ $M(1-M) r_{k}^{2}, U\left(Z_{k}\right) \rightarrow U^{*} \neq 0$ (since $n>2$ ), $\left|A\left(Z_{k}\right)\right| \sim \frac{M}{r_{k}^{2}}$, and $\left|Q\left(Z_{k}\right)\right| \sim M r_{k}$. Hence $\lambda\left(Z_{k}\right) \sim \frac{U^{*}}{M(1-M) r_{k}^{2}}$ and $\left|\lambda\left(Z_{k}\right) Q\left(Z_{k}\right)\right| \sim \frac{U^{*}}{(1-M) r_{k}}$. Thus when $k$ is large $\left|A\left(Z_{k}\right)\right|<\left|\lambda\left(Z_{k}\right) Q\left(Z_{k}\right)\right|$. Since this contradicts the centrality of $G\left(Z_{k}\right)$, condition iii in conjunction with the centrality of the configurations $G\left(Y_{i}\right)$ is impossible.

One-point isolation can be viewed as a consequence of each of the remaining theorems in the paper. These are of interest apart from the theorem. They capture more completely the results that led to it and ultimately to the proof of Theorem 6.2

In Theorems 6.3 6.4, 6.5, and 6.6 the notation and assumptions of Definition 4.1 will be used. If $F(X): R^{2 n} \rightarrow R^{2}$ and $T \in R^{2}$ is a unit vector, we define the directional derivative at $X^{0}$ of $F$ with respect to $X_{j}$ in the direction $T$ to be $\operatorname{Lim}_{h \rightarrow 0^{+}} \frac{F\left(X^{0}+h T_{j}^{0}\right)-F\left(X^{0}\right)}{h}$. It will be denoted by $D_{j, T} F\left(X^{0}\right)$.

Theorem 6.3. If $n>4$ and the planar configuration $\left\{X_{10}, X_{20}, \ldots, X_{n 0} ; m_{1}, m_{2}\right.$, $\left.\ldots, m_{n}\right\}$ is central, then for any $k, 1 \leq k \leq n$, and any unit vector $T \in R^{2}$, there exist at most three distinct values of $j, 1 \leq j \leq n$, and different from $k$, for which $D_{k, T} P_{j}\left(X^{0}\right)=0$. 
Proof. For consistency with previous results we begin by reindexing the particles so that the $k$ th is denoted as the $n$th and the $n$th as the $k$ th. So with no loss we need only establish the result for $k=n$.

Case i: $X_{n 0}$ is not the center of mass.

In is case there is a transformation $H$ which transforms $\left\{X_{10}, X_{20}, \ldots, X_{n 0} ; m_{1}\right.$, $\left.m_{2}, \ldots, m_{n}\right\}$ to zero-one form. $H$ consists of a translation which moves $X_{n 0}$ to the origin, followed by a rotation $R$ about the origin which puts the center of mass of the translated configuration on the positive $x$ axis, and then finally followed by a scale change which multiplies distances by a factor of $c>0$ and results in the center of mass being located at $\left[\begin{array}{l}1 \\ 0\end{array}\right]$. Using $\widetilde{X}_{j}$ to denote $H\left(X_{j}\right)$, it follows that $\widetilde{X}_{n 0}=0$ and the configuration $\left\{\widetilde{X}_{10}, \widetilde{X}_{20}, \ldots, \widetilde{X}_{n 0} ; m_{1}, m_{2}, \ldots, m_{n}\right\}$ is central and in zero-one form. The transformation also causes $X^{0}+h T_{n}^{0}$ to be transformed to $\widetilde{X}^{0}+\tilde{h} \widetilde{T}_{n}^{0}$, where $\widetilde{X}^{0}$ is obtained from $X^{0}$ by replacing $X_{i 0}$ by $\widetilde{X}_{i 0}, \widetilde{T}=R(T)$, and $\tilde{h}=c h$.

Since $\left|P_{j}\left(\widetilde{X}^{0}+\tilde{h} \widetilde{T}_{n}^{0}\right)\right|=\frac{1}{c^{2}}\left|P_{j}\left(X^{0}+h T_{n}^{0}\right)\right|$, if $j<n$ and $D_{n, T} P_{j}\left(X^{0}\right)=0$, then $0=\operatorname{Lim}_{h \rightarrow 0^{+}} \frac{P_{j}\left(X^{0}+h T_{n}^{0}\right)-P_{j}\left(X^{0}\right)}{h}=\operatorname{Lim}_{h \rightarrow 0^{+}} \frac{P_{j}\left(X^{0}+h T_{n}^{0}\right)}{h}$, and consequently $\operatorname{Lim}_{\tilde{h} \rightarrow 0^{+}} \frac{\left|P_{j}\left(\widetilde{X}^{0}+\tilde{h} \widetilde{T}_{n}^{0}\right)\right|}{\tilde{h}}=\frac{1}{c^{3}} \operatorname{Lim}_{h \rightarrow 0^{+}} \frac{\left|P_{j}\left(X^{0}+h T_{n}^{0}\right)\right|}{h}=0$. From Corollary [5.1, there are at most three distinct $j$ less than $n$ for which $\operatorname{Lim}_{\tilde{h} \rightarrow 0^{+}} \frac{\left|P_{j}\left(\widetilde{X}^{0}+\tilde{h} \widetilde{T}_{n}^{0}\right)\right|}{\tilde{h}}=0$. Hence there are at most three distinct $j$ less than $n$ for which $D_{n, T} P_{j}\left(X^{0}\right)=0$.

Case ii: $X_{n 0}$ is the center of mass.

As in Case i, if $j<n$ and $D_{n, T} P_{j}\left(X^{0}\right)=0$, then $\operatorname{Lim}_{h \rightarrow 0^{+}} \frac{\left|P_{j}\left(X^{0}+h T_{n}^{0}\right)\right|}{h}=0$. From Corollary 4.1. there are at most two distinct $j$ less than $n$ for which this limit can hold. Hence there are at most two distinct $j$ less than $n$ for which $D_{n, T} P_{j}\left(X^{0}\right)=0$. This completes the proof.

Selecting four of the $n$ particles for special consideration, which with no loss we assume to be the first four, we define

$$
P(X ; 4)=\left[\begin{array}{l}
P_{1}(X) \\
P 2(X) \\
P_{3}(X) \\
P_{4}(X)
\end{array}\right] .
$$

$P(X)$ will be defined by

$$
P(X)=\left[\begin{array}{c}
P_{1}(X) \\
P_{2}(X) \\
\vdots \\
P_{n}(X)
\end{array}\right]
$$

$D P\left(X^{0}\right)$ will denote the $2 n \times 2 n$ derivative matrix of $P(X)$ evaluated at $X^{0}$ and $D P\left(X^{0} ; 4\right)$ the $8 \times 2 n$ derivative matrix of $P(X ; 4)$ evaluated at $X^{0}$. In each of the derivative matrices, columns $2 k-1$ and $2 k$ correspond respectively to differentiation with respect to $x_{k}$ and $y_{k}$, where $X_{k}=\left[\begin{array}{l}x_{k} \\ y_{k}\end{array}\right]$.

Theorem 6.4. Whenever $n>4, k>4$, and the planar configuration $\left\{X_{10}, X_{20}\right.$, $\left.\ldots, X_{n 0} ; m_{1}, m_{2}, \ldots, m_{n}\right\}$ is central, columns $2 k-1$ and $2 k$ of the derivative matrix $D P\left(X^{0} ; 4\right)$ are independent. 
Proof. If they were not independent, there would exist a unit vector $T$ such that $D P\left(X^{0} ; 4\right) T_{k}^{0}=0$. This would imply $D_{k, T} P_{j}\left(X^{0}\right)=0, j=1,2,3,4$, contradicting Theorem [6.3.

Theorem 6.5. For $1 \leq k \leq n$, whenever $n>4$ and the planar configuration $\left\{X_{10}, X_{20}, \ldots, X_{n 0} ; m_{1}, m_{2}, \ldots, m_{n}\right\}$ is central, columns $2 k-1$ and $2 k$ of the derivative matrix $D P\left(X^{0}\right)$ are independent.

Proof. Choose $k$ and reindex the configuration so that $k$ th mass becomes the fifth mass. It follows from the previous theorem that in the derivative matrix associated with the reindexing the subcolumns consisting of the upper 8 rows of the 9 th and 10 th columns are independent and hence the entire columns are independent. Thus columns $2 k-1$ and $2 k$ of the original derivative matrix $D P\left(X^{0}\right)$ are independent.

The final theorem states that a slight perturbation in the position of any single point-mass in a planar central configuration of more than 4 points destroys the centrality property for at least $n-4$ of the nonperturbed masses.

Theorem 6.6. There exists a disk centered at each point mass in a planar central configuration of more than 4 points such that if that mass alone is perturbed and moved from the center of the disk to some other point in the disk, then the centrality property is destroyed for at least $n-4$ of the unperturbed masses.

Proof. Let the central configuration be $\left\{X_{10}, X_{20}, \ldots, X_{n 0} ; m_{1}, m_{2}, \ldots, m_{n}\right\}$. Assume the mass to be perturbed is located at $X_{n 0}$. If such a disk did not exist, there would exist a sequence $\left\{T_{i}\right\}$ of unit vectors in $R^{2}$ and sequences $\left\{h_{i}\right\}$ and $\left\{j_{i}, k_{i}, l_{i}, n_{i}\right\}$ such that i) $h_{i} \rightarrow 0$, ii) $1 \leq j_{i}<k_{i}<l_{i}<n_{i}<n$, and iii) when the mass located at $X_{n 0}$ is moved to $X_{n 0}+h_{i} T_{i}$ the unperturbed masses located at $X_{j_{i}}, X_{k_{i}}, X_{l_{i}}, X_{n_{i}}$ possess the centrality property. By considering convergent subsequences, it can be assumed that $T_{i} \rightarrow T$, and $j_{i}=j_{1}, k_{i}=k_{1}, l_{i}=i_{1}$, and $n_{i}=n_{1}$. With

$$
T_{i, n}^{0}=\left[\begin{array}{c}
X_{10} \\
X_{20} \\
\vdots \\
X_{j 0} \\
\vdots \\
X_{n 0}+T_{i}
\end{array}\right]-X^{0},
$$

we have $0=P_{j_{1}}\left(X^{0}+h_{i} T_{i, n}^{0}\right)=P_{j_{1}}\left(X^{0}+h_{i} T_{i, n}^{0}\right)-P_{j_{1}}\left(X^{0}\right)=D P_{j_{1}}\left(X^{0}\right) h_{i} T_{i, n}^{0}$ $+o\left(h_{i}\right)=D P_{j_{1}}\left(X^{0}\right) h_{i} T_{n}^{0}+D P_{j_{1}}\left(X^{0}\right) h_{i}\left(T_{i, n}^{0}-T_{n}^{0}\right)+o\left(h_{i}\right)$. Consequently, $0=$ $D P_{j_{1}}\left(X^{0}\right) T_{n}^{0}+\varepsilon_{i}$, where $\varepsilon_{i} \rightarrow 0$. Thus $0=D P_{j_{1}}\left(X^{0}\right) T_{n}^{0}=D_{n, T} P_{j_{1}}\left(X^{0}\right)$. Since this is also true when $j_{1}$ is replaced by either $k_{1}, l_{1}$, or $n_{1}$, we arrive at a contradiction based upon Theorem 6.3.

\section{REFERENCES}

1. G. Buck, On clustering in central configurations, Proc. Amer. Math. Soc. 108 (1990), 801-810. MR 90f:70016

2. L. Euler, De moto rectilineo trium corporum se mutuo attahentium, Novi Comm. Acad. Sci. Imp. Petrop. 11 (1767), 144-151.

3. J. L. Lagrange, Ouvres, Volume 6, Paris, 1873, 272-292.

4. K. R. Meyer, and G. R. Hall, Introduction to Hamiltonian Dynamical Systems and the N-Body Problem, Springer-Verlag, 1992. MR 93b:70002 
5. R. Moeckel, On central configurations, Math. Z. 205 (1990), 499-517. |MR 96d:70015]

6. R. Moeckel, Relative equilibria of the four-body problem, Ergodic Theory and Dynamical Systems 5 (1985), 417-435. MR 87b:70011

7. F. R. Moulton, The straight line solutions of the problem of $N$ bodies, Ann. Math., II. Ser. 12, 1910, 1-17.

8. D. Saari, On the role and properties of $n$ body central configurations, Celest. Mech. 21 (1980), 9-20. MR 81a:70016

9. S. Smale, Mathematical problems for the next century, The Mathematical Intelligencer 20 (1998), 7-15. MR 99h:01033

Department of Mathematics, Saint Anselm College, Manchester, New Hampshire 03102 